\title{
Photodetectors for Scintillator Proportionality Measurement
}

*William W. Moses ${ }^{1}$, Woon-Seng Choong ${ }^{1}$, Giulia Hull ${ }^{2}$, Steve Payne ${ }^{2}$, Nerine Cherepy ${ }^{2}$,

\author{
and John D. Valentine ${ }^{2}$ \\ ${ }^{1}$ Lawrence Berkeley National Laboratory \\ ${ }^{2}$ Lawrence Livermore National Laboratory
}

*This work is supported in part by the National Nuclear Security Administration, Office of Defense Nuclear Nonproliferation, Office of Nuclear Nonproliferation Research and Engineering (NA-22) of the U.S. Department of Energy under contract No. DE-AC0205CH11231, grant number NNSA LB06-316-PD05 / NN2001000, and in part under the auspices of the U.S. Department of Energy Lawrence Livermore National Laboratory under Contract DE-AC52-07NA27344 and by the Domestic Nuclear Detection Office of the Department of Homeland Security.

Corresponding Author: William W. Moses (wwmoses@lbl.gov, ++1-510-486-4432,++1510-486-4768 FAX)

\footnotetext{
Abstract

We evaluate photodetectors for use in a Compton Coincidence apparatus designed for measuring scintillator proportionality. There are many requirements placed on the photodetector in these systems, including active area, linearity, and the ability to accurately measure low light levels (which implies high quantum efficiency and high signal-to-noise ratio). Through a combination of measurement and Monte Carlo simulation, we evaluate a number of potential photodetectors, especially photomultiplier
} 
tubes and hybrid photodetectors. Of these, we find that the most promising devices available are photomultiplier tubes with high $(\sim 50 \%)$ quantum efficiency, although hybrid photodetectors with high quantum efficiency would be preferable.

Keywords \& PACS

Scintillator nonproportionality, photodetectors, photomultiplier tubes, hybrid photodetectors.

29.40.Mc, $85.60 . \mathrm{Ha}, 85.60 . \mathrm{Gz}$

1. Introduction

The Compton Coincidence Technique [1-3] is commonly used to understand scintillator proportionality. Measurements of scintillator proportionality are important, as deviations from proportionality degrade the energy resolution for a scintillator material [4-10]. The Compton Coincidence Technique measures the electron response- the dependence of the scintillator luminosity (photons / MeV) on the energy of the quanta (in this case, electrons) that excite it. While the Compton Coincidence apparatus we have developed is described in detail in [11,12], the basic technique is to have a monoenergetic gamma ray from an isotopic source (usually $662 \mathrm{keV}$ emissions from Cs137) undergo Compton scatter within a scintillator sample. The energy of the scattered gamma ray is measured by a high-purity germanium (HPGe) detector, and the amount of energy deposited in the scintillator is given by the difference between the initial and scattered gamma ray energies. The scintillator is coupled to a photodetector that measures the amount of light produced by this interaction, and so allows the scintillator luminosity to be calculated at a variety of energies. A sample electron response measurement is 
shown in figure 1a. The underlying measurement for each of the data points in figure 1a, which is the pulse height distribution recorded by the photomultiplier tube (PMT) when the scintillator is excited with monoenergetic Compton electrons, is shown in figure $1 \mathrm{~b}$ for three different electron energies.

The requirements placed on the photodetector in these systems are quite stringent. In general, one needs to accurately measure the light produced by a scintillator crystal (our "standard" scintillator geometry is a right circular cylinder that is 0.5 inches diameter and 0.5 inches tall) that is excited by electrons with anywhere between $1 \mathrm{keV}$ and $662 \mathrm{keV}$ of energy. Thus, the active area must be larger than $\sim 1$ square centimeter in order to efficiently collect light from the scintillator sample. The response must be linear $(<0.5 \%$ deviation is needed) over a dynamic range extending up to $\sim 50,000$ incident photons. Single photoelectron resolution is desired, as is high quantum efficiency, as these properties make accurate determination of the luminosity at low $(\sim 1 \mathrm{keV})$ excitation energies possible (see figure 1b). The physics of proportionality makes measuring the luminosity at these low energies particularly important, but the low total light output (1 $\mathrm{keV}$ of energy deposited in NaI:Tl typically results in approximately 30 scintillation photons incident on the photodetector) makes accurate measurement particularly challenging.

We evaluate several types of photodetectors for this purpose. Despite their excellent linearity and quantum efficiency, we rule out PIN photodiodes and avalanche photodiodes because their signal-to-noise ratio is inadequate $\left(1 \mathrm{~cm}^{2}\right.$ area devices are unable to resolve single photoelectrons). We also rule out silicon photomultipliers (SiPMs) [13] as their active area is generally too small and their linearity is usually quite 
poor over the wide dynamic range required for this application. Thus we consider three types of photodetector-conventional bialkali PMTs, bialkali PMTs with enhanced quantum efficiency ( $35 \%$ to $50 \%$, as opposed to $25 \%$ ) $[14,15]$, and hybrid photodetectors (HPDs) [16-20]. An HPD is similar to a conventional PMT except that the gain is provided not by a dynode structure, but by accelerating the photoelectrons to $\sim 15 \mathrm{keV}$ and then having these electrons deposit their kinetic energy in a silicon PIN diode where the charge is multiplied by impact ionization.

\section{Linearity}

One advantage of the HPD is that the device is intrinsically very linear. The nonlinearities in conventional PMTs arise from the dynode structure, where the "cloud" of multiplied electrons can both alter the current (and thus the voltage and therefore the gain) in the resistor chain that biases the dynode chain and also produce space-charge effects that shield some of the electrons in the cloud (and thus reduce their gain) [21,22]. The HPD does not have this dynode structure and instead is linear as long as the mean ionization energy in silicon remains constant (3.64 eV per e/h pair [23]). In addition, the one-step multiplication process results in much lower gain fluctuations than those in PMTs.

With proper care taken in the dynode design and the biasing circuit, conventional PMTs can also be extremely linear, but it is important to check their performance. We therefore measure the linearity of four conventional PMTs and one PMT with a high quantum efficiency photocathode. The linearity was measured using the apparatus shown in figure 2 and a modification of the technique described in [24]. The pulser creates a 10 
ns wide electrical pulse that creates a 20 ns wide flash of light from either or both of the blue LEDs shown in figure 2, and the amount of light produced in each LED is controlled by a resistor. Both LEDs are optically coupled into a 1-inch diameter Lucite sphere that has a 0.25 -inch diameter, 2-inch long Lucite rod glued to it. All surfaces of the Lucite sphere and rod are wrapped with white Teflon tape, except for the end of the rod. This Lucite assembly acts as a light mixer / diffuser, and also transports the light emitted by the LEDs through a neutral density filter toward the PMT.

The procedure for measuring the linearity begins by pulsing the upper LED. Throughout the entire procedure, the resistor value for the upper LED is never changed, and so this LED always produces the same amount of light, which we define as one unit of light. The PMT output is recorded by a pulse height analyzer, histogrammed, and the centroid of the distribution computed. The upper LED is turned off, the lower LED is pulsed, and its resistor adjusted until the resulting centroid lies at the same position as when the upper LED was being pulsed. At this point, it is also producing one unit of light (within measurement error, which is $\sim 0.1 \%$ ). Both LEDs are then pulsed simultaneously, so that two units of light impinge on the PMT. The centroid of the resulting pulse height distribution is computed, and represents the response of the PMT to two units of light. The top LED is then turned off, and the resistor for the lower LED readjusted so that it has the same centroid, and so now produces two units of light. The top LED is turned on again and both LEDs pulsed simultaneously, so three units of light impinge on the PMT. The centroid is measured, the top LED turned off, the resistor for the bottom LED readjusted, and the process continues until the PMT has been pulsed with 11 evenly spaced amplitudes of light. The maximum amplitude is roughly 2.5 times the light output 
of $13 \mathrm{~mm}$ diameter, $13 \mathrm{~mm}$ tall right circular cylinder of $\mathrm{LaBr}_{3}: \mathrm{Ce}$ excited by $662 \mathrm{keV}$ gamma rays, which corresponds to approximately 100,000 photons impinging on the PMT. Note that the process of adding two light pulses together ensures that all 11 light amplitudes are evenly spaced, even if the response of the PMT is non-linear. The linearity of the PMT is then determined by plotting the centroid of the pulse height as a function of the light amplitude.

Figure 3 shows the deviation from linearity for several different PMTs. The deviation is calculated by fitting the response to the lowest three light amplitudes to a line that goes through the origin, then plotting the percentage difference between the measured value and this line. The Photonis XP2060B is linear $(<0.4 \%$ deviation $)$ over the entire range when operated at its nominal bias voltage of $800 \mathrm{~V}$, the Hamamatsu R6231 has similar linearity when operated at $800 \mathrm{~V}$, as does a high quantum efficiency version of the R6231 (the SBA version, which has $\sim 35 \%$ quantum efficiency). However, the R6231 has $\sim 6 \%$ non-linearity when operated at its nominal voltage of 1200 V. The Hamamatsu R7724 has $\sim 3 \%$ non-linearity when operated at its nominal voltage of $1300 \mathrm{~V}$, while the Hamamatsu R1306 has $>25 \%$ non-linearity when operated at its nominal voltage of 1200 V. The Photonis XP2060B was the conventional PMT of choice, as it simultaneously provides good linearity and high gain, and was used to collect the data presented in figure 1.

\section{Small Signal Response}

The electron response data shown in figure 1 indicates that the errors are largest at low excitation energies and the most important physics also occurs in this region (the 
largest sample to sample differences occur in this energy region). We use a two-step Monte Carlo simulation to predict whether we can improve the low-energy performance of the apparatus for NaI:Tl scintillator. For the first step we use the GEANT4 simulation package [25] to model the initial gamma ray interactions in the scintillator and subsequent detection by the HPGe detectors. The simulation includes Compton and photoelectric interactions in the collimator, scintillator, and HPGe detectors, as well as Xray fluorescence and Auger electron production in the scintillator. The outputs from this portion of the simulation are the "true" amount of energy deposited in both the scintillator and each of the HPGe detectors.

The second part of the simulation models the response of the detectors - modeling scintillator and photodetector physics as well as finite energy resolution in the HPGe detectors. For each event the conversion of energy deposited in the scintillator into light is done using a parameterized version of the electron response curve shown in figure 1a (conversion of energy into scintillation photons), assuming a 75\% collection efficiency (probability that an emitted scintillation photon impinges on the photodetector), and then using the photodetector quantum efficiency (probability that an impinging photon creates a photoelectron) to find the mean number of photoelectrons produced for this energy deposit. Event-to-event statistical fluctuations in the number of photoelectrons produced by the photodetector are introduced by randomly choosing an integer number of resulting photoelectrons from a Poisson distribution with the appropriate width. The photodetector response was then modeled by assuming two components - a single photoelectron response and electronic noise. The response to multiple $(n)$ photoelectrons is simulated by convolving the response of $n$ single photoelectrons and the electronic noise. The 
values for the single photoelectron response and electronic noise are obtained by fitting to data published in $[20,26]$. While the simulation of the number of photoelectrons produced is somewhat simplistic, it is adequate for this investigation. The "task" that the photodetector must perform is to accurately measure the number of photons that impinges on it, so while it is critical that the number of incident photons be known (and be representative of the anticipated number of incident photons), detailed simulation of the incident photon flux is unnecessary.

Figure 4 shows both the modeled and true photoelectron response for a conventional PMT (a Burle 8850) [26] and an HPD (DEP/Photonis PP0275C) [20]. The modeled and true distributions are very similar for both photodetectors. However, the HPD distribution consists of narrow peaks on top of a broad background, and the shape of the broad backgrounds are slightly different. This is due to the simplistic simulation done in this work - more accurate agreement can be obtained using a more sophisticated simulation of the HPD single photoelectron response [27].

Figure 5 shows the simulated response (similar to that shown in figure 1b) for four different photodetectors-conventional PMTs and HPDs with conventional (25\% QE) and high efficiency $(50 \% \mathrm{QE})$ photocathodes. The benefit of increased quantum efficiency is readily apparent, as the position of the centroid is a factor of two higher with the high QE photocathodes. The individual photoelectron structure can also be seen (at low electron energies) in the HPDs. These data are analyzed as if they were real proportionality data, and the "measured" electron response computed. The bias (difference between the "measured" electron response and the input to the simulation) and the variance (run to run difference in the "measured" response) are computed and 
shown in figure 6.

Figure 6 shows that there is a significant reduction in both the bias and the variance at low electron energies when a PMT with higher quantum efficiency is used. This is due to the increased signal (number of photoelectrons) and so better signal-to-noise ratio produced by the higher QE photocathode. It perhaps is surprising that the HPD, with its outstanding single photoelectron resolution and ability to count photoelectrons, does not improve the performance. This is due to the fact that the analysis presently makes no attempt to count the number of photoelectrons in the distributions displayed in figure 5 and fit to a Poisson distribution. Instead, it merely takes the mean of the distribution. However, fitting to a Poisson distribution is difficult due to the tails seen in the HPD spectra shown in figure 4. These tails account for a significant fraction of the events in the distribution, so the tails must be modeled extremely well to prevent biasing the fit. While this is possible (reference [27], for example, describes a more accurate method for modeling the HPD response), considerable care should be taken to validate a fitting method based on "photoelectron-counting" the HPD data and to estimate the fitting error.

\section{Conclusion}

We analyze the photodetector requirements for a Compton Coincidence apparatus that is used to measure scintillator proportionality. While linearity is quite important, many photodetectors (notably conventional PMTs and HPDs) have sufficient linearity, provided that they are selected and operated carefully. Small signal detection is also important, and high quantum efficiency versions of either conventional PMTs or HPDs can dramatically improve the low energy performance of the instrument. The ability of 
HPDs to count single photoelectrons is also likely to improve the low energy performance, but to do so requires accurate modeling of the HPD single photoelectron response, which is possible but non-trivial [27].

Thus, replacing the conventional PMT with a PMT having high quantum efficiency provides a relatively easy way to improve performance. Replacing the conventional PMT with an HPD is likely to also improve performance, albeit with somewhat longer development time. The most attractive option is an HPD with high quantum efficiency, as they would combine the advantages of both higher signal and the ability to count photons. 


\section{References}

[1] J.D. Valentine, et al. Nucl. Instr. Meth. A-353 (1994) 37-40.

[2] B.D. Rooney, et al. IEEE Trans. Nucl. Sci. NS-43 (1996) 1271-1276.

[3] B.D. Rooney, Ph. D. Thesis, University of Cincinnati (1996).

[4] C.D. Zerby, et al. Nucl. Instr. Meth. 12 (1961) 115-123.

[5] J.R. Prescott, et al. Nucl. Instr. Meth. 39 (1969) 51-55.

[6] P. Dorenbos, et al. IEEE Trans. Nucl. Sci. NS-42 (1995) 2190-2202.

[7] M. Balcerzyk, et al. IEEE Trans. Nucl. Sci. NS-47 (2000) 1319-1323.

[8] M. Moszynski, et al. Nucl. Instr. Meth. A-484 (2002) 259-269.

[9] E.V.D. van Loef, et al. IEEE Trans. Nucl. Sci. NS-50 (2003) 155-158.

[10] W.W. Moses, et al. IEEE Trans. Nucl. Sci. NS-55 (2008) 1049-1053.

[11] W.S. Choong, et al. IEEE Trans. Nucl. Sci. NS-55 (2008) 1753-1758.

[12] W.S. Choong, et al. IEEE Trans. Nucl. Sci. NS-55 (2008) 1073-1078.

[13] P. Buzhan, et al. Nucl. Instr. Meth. A-504 (2003) 48-52.

[14] M. Suyama. Proceedings of Science PD07 (2007) 018.

[15] M. Kapusta, et al. IEEE NSS/MIC Conference Record (ed. B. Yu) N06-2 (Honolulu, HI, 2007).

[16] C.P. Datema. Nucl. Instr. Meth. A-387 (1997) 100-103.

[17] C. D'Ambrosio, et al. Nucl. Instr. Meth. A-434 (1999) 387-398.

[18] C. D'Ambrosio, et al. IEEE Trans. Nucl. Sci. NS-47 (2000) 6-12.

[19] C. D'Ambrosio, et al. Nucl. Instr. Meth. A-501 (2003) 463-498.

[20] M. Moszynski, et al. J. Inst. 1 (2006) P05001.

[21] D.R. Carter. Photomultiplier Handbook (Burle Industries, Lancaster, PA, 1980).

[22] S.-O. Flyckt, et al. Photomultiplier Tubes - Principles and Applications (Photonis Imaging

Sensors, Brive, France, 2002).

[23] F. Scholze, et al. Proc. SPIE 2808 (1996) 534-543.

[24] M. Vicic, et al. Nucl. Instr. Meth. A-507 (2003) 636-642.

[25] S. Agostinelli, et al. Nucl. Instr. Meth. A-506 (2003) 250-303.

[26] G. Zambra, et al. Rev. Sci. Instr. 75 (2004) 2762-2765.

[27] T.T. De Fatis. Nucl. Instr. Meth. A-385 (1997) 366-370. 


\section{Figure Captions}

Figure 1: a) Plot of the NaI:Tl electron response (light output when excited by electrons, normalized to the value at $444 \mathrm{keV}$ ), as measured with a Compton Coincidence device. b) Raw data used to create some of the individual data points in figure 1a. These are histograms of the light output measured by the PMT when the NaI:Tl crystal is excited with $1.25,3.25$, and $5.25 \mathrm{keV}$ electrons.

Figure 2. Diagram of the apparatus used to measure the photomultiplier tube linearity.

Figure 3. Plot of the deviation from linearity as a function of light intensity for several photomultiplier tubes.

Figure 4. Comparison of pulse height distributions of photodetectors excited at low light levels. The upper row is published data from [26] (conventional PMT, on the left) and [20] (HPD, on the right). The lower row has corresponding results from the Monte Carlo simulation used in this work.

Figure 5. Simulated pulse height distributions (similar to those shown in figure 1b) using different assumptions for the photodetector used. The left-hand plot assumes $1.25 \mathrm{keV}$ electron excitation energy, the center plot assumes $3.25 \mathrm{keV}$, and the right hand plot assumes $5.25 \mathrm{keV}$. 
Figure 6 . The bias and variance in the electron response measurement as a function of electron energy for different assumptions regarding the photodetector used. 


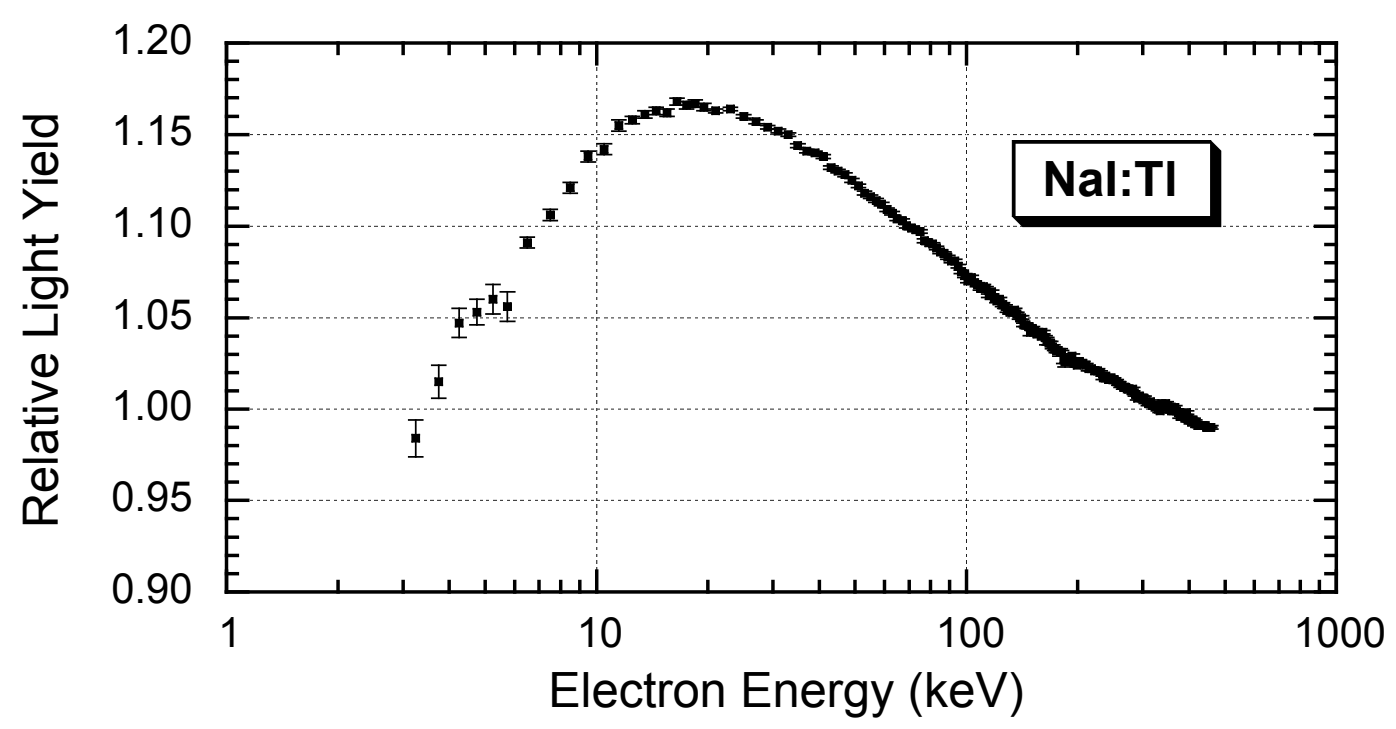

Figure 1a

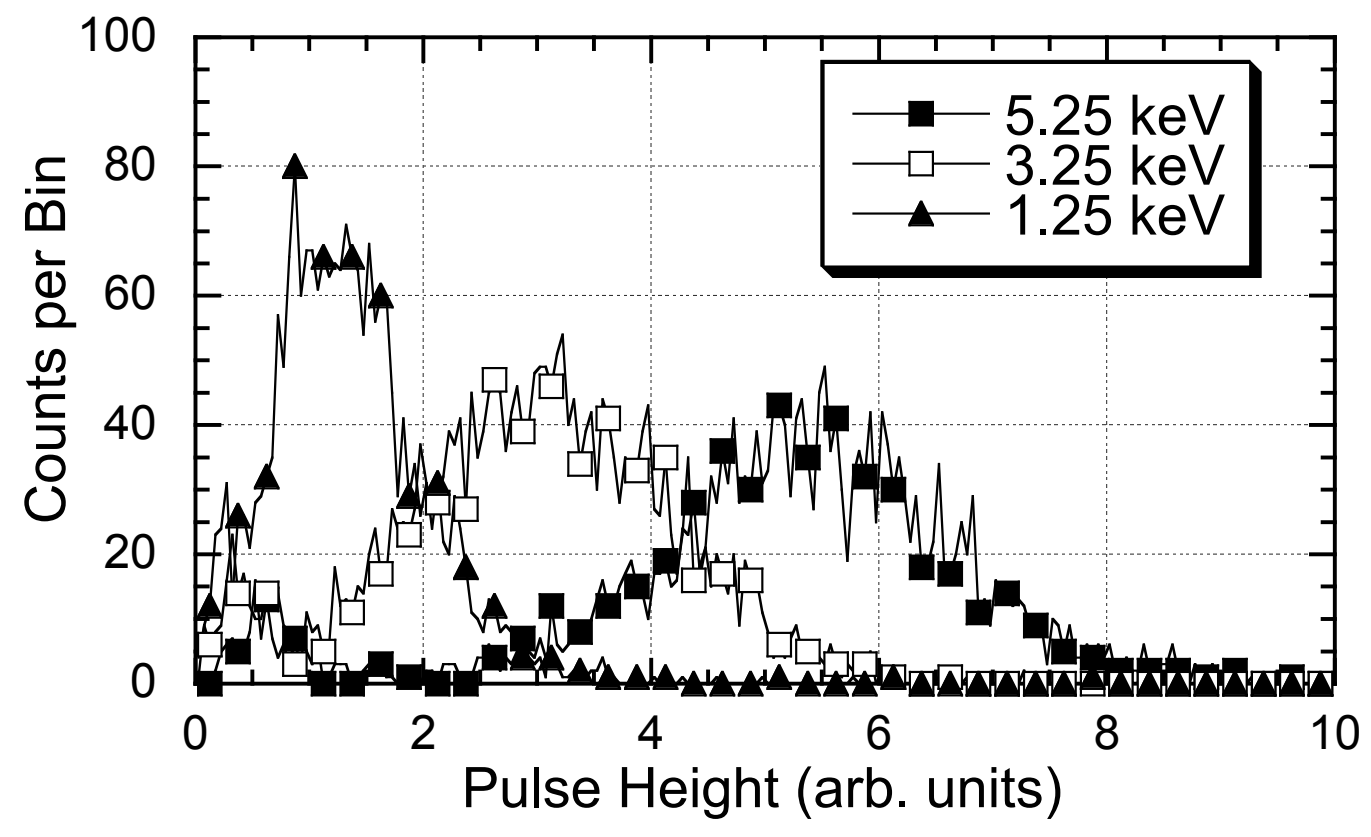

Figure $1 \mathrm{~b}$ 


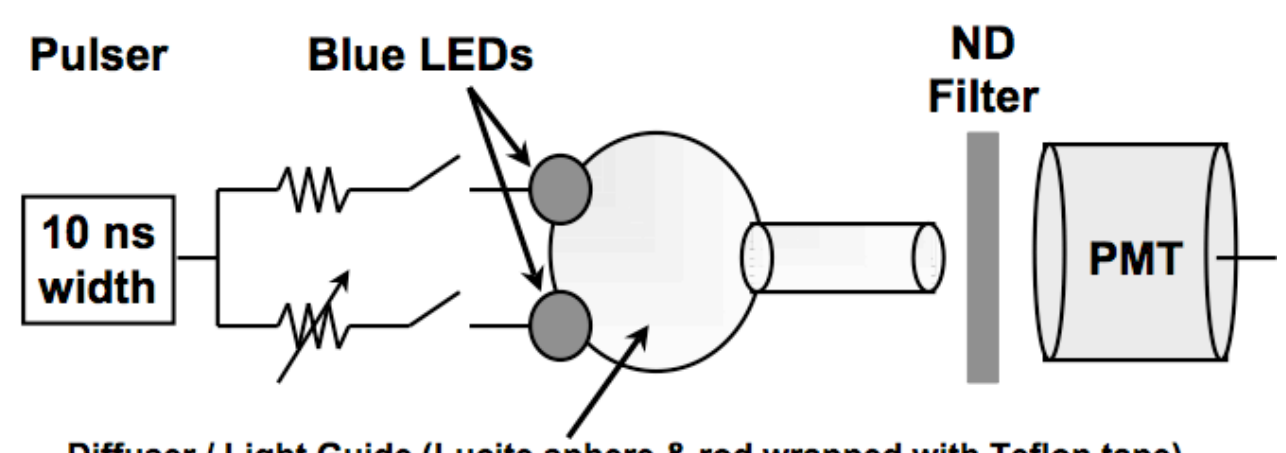

Diffuser / Light Guide (Lucite sphere \& rod wrapped with Teflon tape)

Figure 2

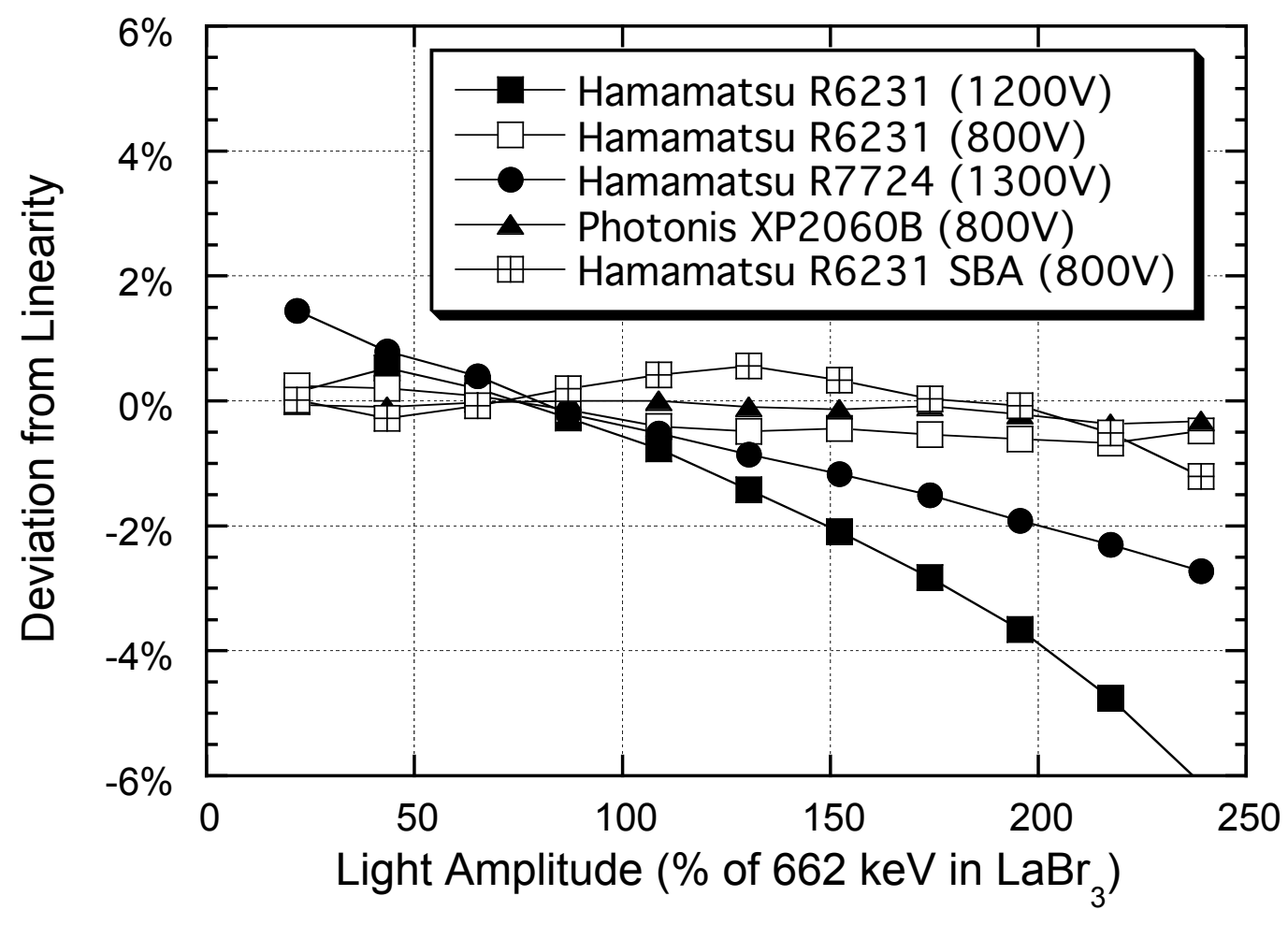

Figure 3 

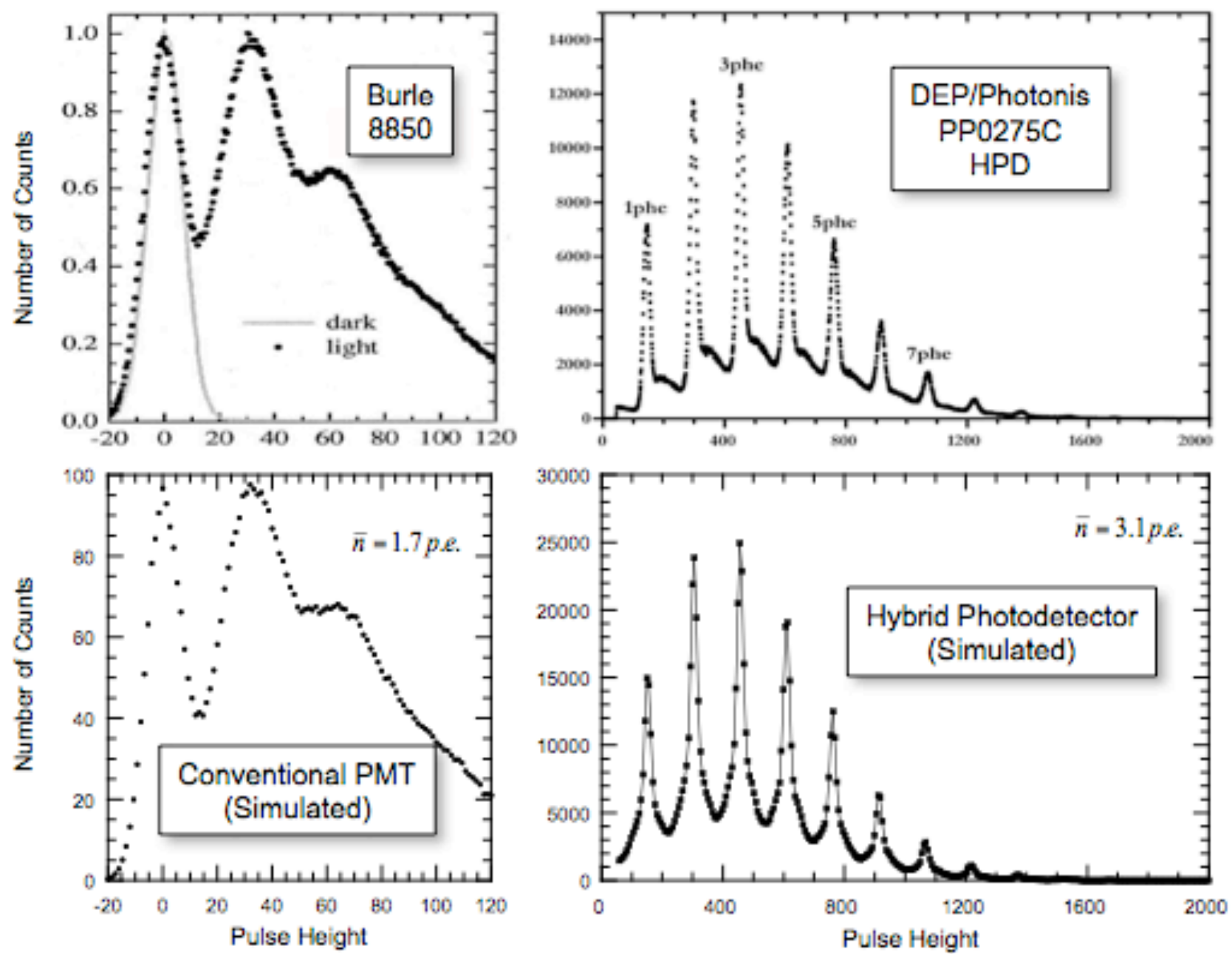

Figure 4 

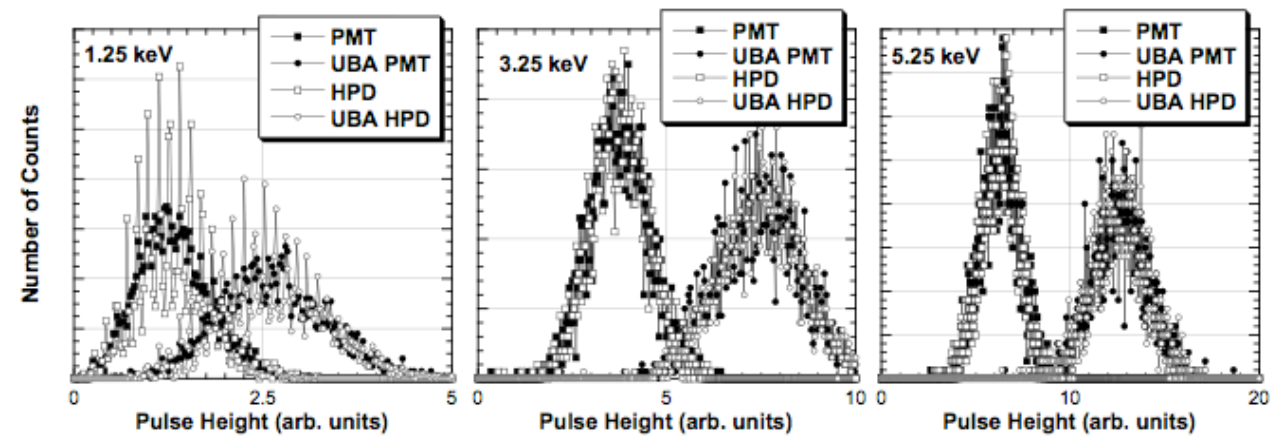

Figure 5
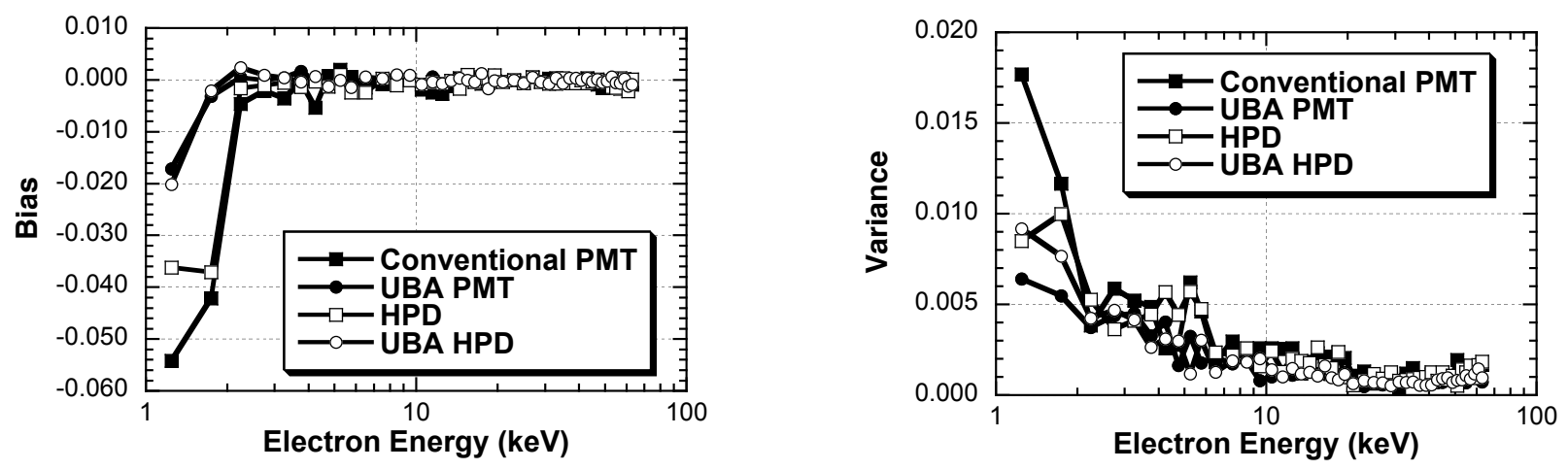

Figure 6 


\section{LEGAL DISCLAIMER}

This document was prepared as an account of work sponsored by the United States Government. While this document is believed to contain correct information, neither the United States Government nor any agency thereof, nor The Regents of the University of California, nor any of their employees, makes any warranty, express or implied, or assumes any legal responsibility for the accuracy, completeness, or usefulness of any information, apparatus, product, or process disclosed, or represents that its use would not infringe privately owned rights. Reference herein to any specific commercial product, process, or service by its trade name, trademark, manufacturer, or otherwise, does not necessarily constitute or imply its endorsement, recommendation, or favoring by the United States Government or any agency thereof, or The Regents of the University of California. The views and opinions of authors expressed herein do not necessarily state or reflect those of the United States Government or any agency thereof or The Regents of the University of California. 